\title{
A MULTI-PARAMETER MINIATURE PHOTOMETRIC ANALYSER FOR FULLY AUTOMATED ENVIRONMENTAL WATER MONITORING
}

\author{
Daniele Paesani ${ }^{1^{*}}$ \\ ${ }^{1}$ Politecnico di Torino, Corso Duca degli Abruzzi 24, 10129 Torino, Italy \\ *E-mail of corresponding author: daa.paesani@gmail.com
}

\begin{abstract}
Nowadays, the increasing demand for constant in-situ monitoring of clean water, wastewater and sea water requires the deployment of automated platforms capable of on-line autonomous sampling, extended unattended operation and of multianalyte detection. The Micro Loop Injection Analyser ( $\mu L I A)$ system hereby presented relies on integrated additively manufactured opto-fluidic components which allow for increased performance solutions to be implemented cost-effectively and in miniaturised footprints, targeting a wide spectrum of applications which ranges from in-situ environmental monitoring and field water testing to industrial process analysis. Preliminary tests of the $\mu L I A$ unit have shown good reproducibility and stability of the system and a good performance of its self-calibration routines.
\end{abstract}

Keywords: automated analyser, microfluidic, photometer, water analysis

\begin{tabular}{c}
\hline Received: $28.06 .2019 . /$ Accepted: 12.07.2019. \\
Published online: 16.07.2019. \\
\hline
\end{tabular}

Professional paper

\section{INTRODUCTION}

The development of in-situ, capillary, fully automated environmental water monitoring networks is thwarted by the high cost and large footprints of currently available analysers and laboratory instrumentation (Mellander et al. 2015; Perks et al. 2015). The aim of the environmental water pollution monitoring is to gather a data through sampling, in situ, to make a rapid action at some hazardous events (Howden et al. 2010). Recent advances in flow injection analysis (Trojanowicz \& Kołacińska 2016) have shown, during a time frame of approximately four decades, a rapid and dynamic evolution of these technologies and their respective detections methods, increasingly focused on nanotechnology and miniaturization (Mills \& Fones 2012). Furthermore, a current trend has lowered the gap between research and commercial instrumentation: increasingly often, instruments are no longer purchased, but self-designed and self-fabricated ad hoc, directly in the lab. This empowers scientists with a high degree of customisation which well suits today's increasingly specific experiments and applications (Heinz et al. 2012). The hereby presented system relies on integrated additively manufactured opto-fluidic components which allow for increased performance solutions to be implemented cost-effectively and in miniaturised footprints, targeting a wide spectrum of applications which ranges from in-situ environmental monitoring and field water testing to industrial process analysis.

\section{SYSTEM DESCRIPTION}

The Micro Loop Injection Analyser ( $\mu$ LIA) hereby presented is a versatile stand-alone microprocessor (ARM CORTEX M4F) based system which integrates a modular design and is capable of multi-parameter (up to 4 simultaneously) colorimetric and fluorometric detection in a compact footprint $(16 \times 11 \times 10 \mathrm{~cm})$, with small reaction volumes (typ. 400 to $1200 \mu \mathrm{l}$ ), low reagent consumption (5 to $200 \mu \mathrm{l}$ per analysis) and extended unattended operation (Figure 1). It also features auto-dilution, calibration and self-diagnosis routines.

The operation principle of colorimetric analysis is based on the Beer-Lambert optical law, which relates the attenuation of light to the properties of the material through which the light is travelling. Suitable reagents will target specific analytes and react with them to produce coloured compounds. By relying on a monochromatic beam - with a wavelength suitable for the reagent's absorbance spectrum - the light transmission through the sample and thus the colour of the solution - can be assessed before and after the addition of the reagent. In particular, the Beer-Lambert law establishes a linear relationship between the absorbance of the medium and the concentration of the light-absorbing species in the medium. By comparing these measurements against factory calibration curves, the concentration of the target analyte can be determined. 




Figure 1. General layout of the instrument Micro Loop Injection Analyser ( $\mu L I A)$

The system design revolves around an integrated, additively manufactured fluidic block, which handles the injection of sample(s) and reagents into the fluidic circuit. It allows the continuous circulation and monitoring of the reaction mixture through the detector(s). The combination of a versatile analysis setup with the modular and multi-wavelength optical detection system allows a wide range of methods to be implemented with minimal effort, such as phosphate (ascorbic acid/molybdate), nutrients, chlorine (DPD), silica, trace metals and more. Standard solutions are programmatically drawn into the analyser at fixed time intervals to perform the automated calibration routines. Similarly, cleaning buffers are regularly used to wash the fluidics and prevent solid precipitates and/or biofilms from fouling the channels. The mechanical construction allows a complete separation of the electronics from the fluidic sections, for greater reliability and safety in case of leakages.

\section{MANIFOLD AND FABRICATION}

The manifold is fabricated by stereolithography in methacrylate photosensitive transparent resin (Formlabs Clear) using a Formlabs Form 2 printer. It houses 6 solenoid direct-actuation isolation rocker membrane valves (ASCO 260) used for fluid selection, which can be easily replaced in case of obsolescence and/or malfunctioning. The monolithic design integrates embedded custom designed valve seats and fluidic interfaces with a minimised total volume of 400 to $1200 \mathrm{ul}$ and optimised flow paths which enhance washing and mixing. The wetted materials are methacrylate resin in the manifold body, while the valve membranes are either in EPDM (ethylene-propylene diene monomer, ASCO, Figure 2), FKM (fluoroelastomer) or FFKM (perfluoroelastomer). After the printing and post-curing steps, the resin block surface was hydrolysed in order to ease de-bubbling and achieve a small degree of surface passivation and hydrophilicity. 


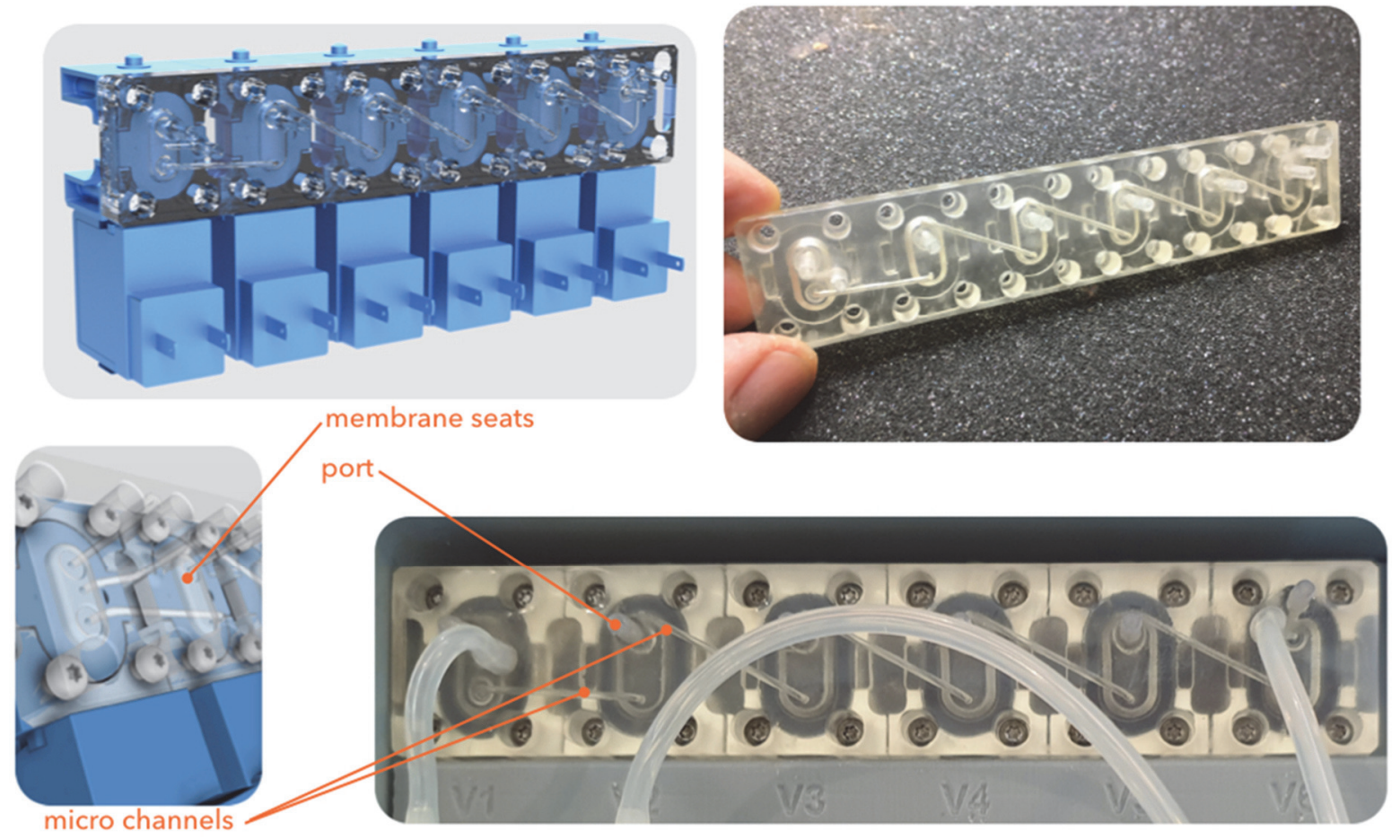

Figure 2. Fluidic block. Top left: CAD model rendering; top right: the block right after printing (supports removed); bottom: channels and valve seat detail. The fluidic channels connecting valve chambers and the inlet/outlet nipples for tubing interconnection are visible.

\section{FLOW-CELL AND OPTICAL DESIGN}

At the heart of any photometric system lies the design of the detection module. The one currently presented is based on a colorimetric transmission measurement mode, realized with a custom SLA printed micro volume flowcell. Some of the most common challenges in photometric systems design are both of a geometrical (optical alignment, source aperture control, intrinsic stray light), environmental (background stray light), electronic (nonlinearity and ageing of the optoelectronic components) and chemical nature (micro-bubbles and fouling).
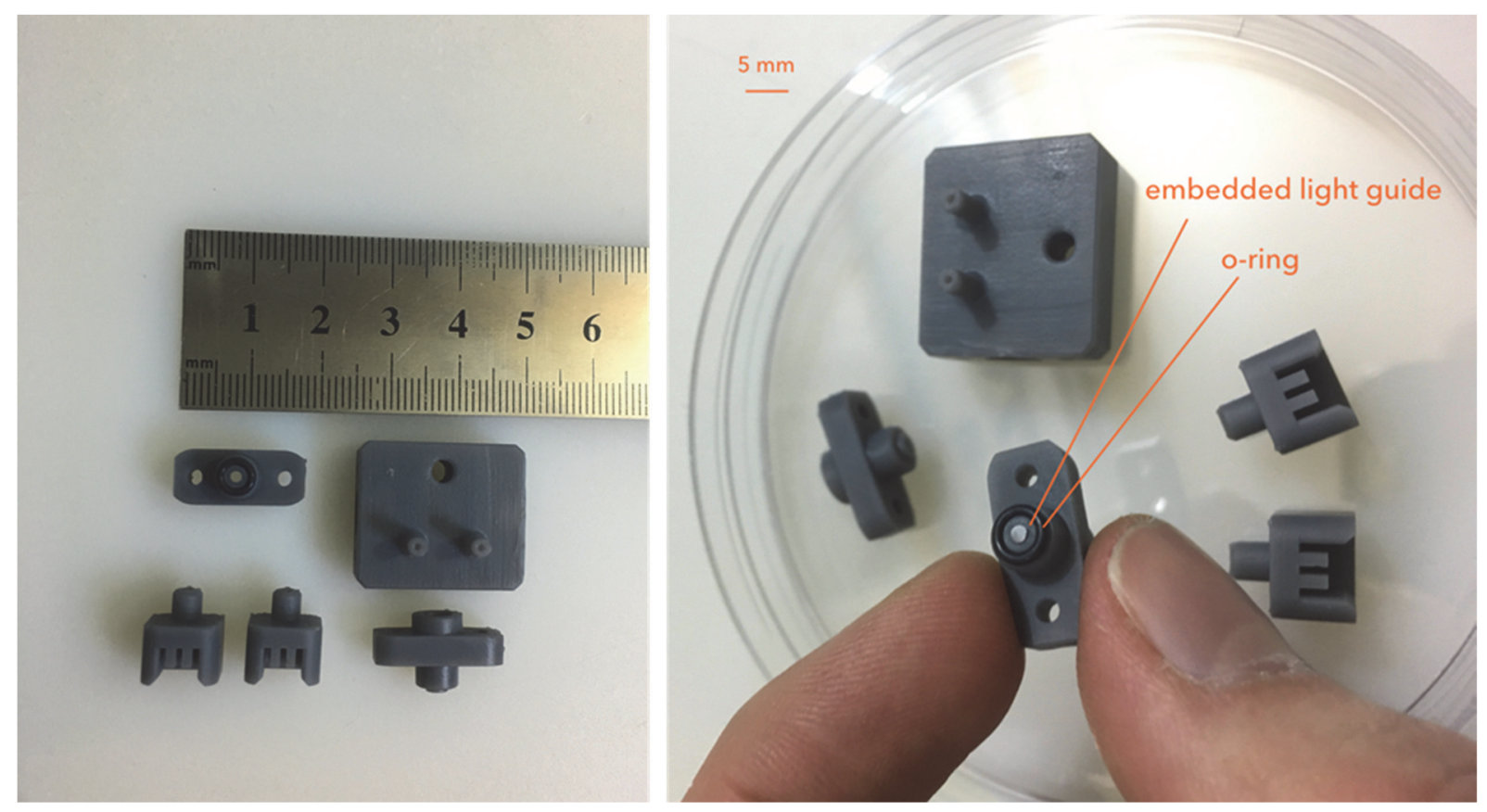

Figure 3. Flow cell components. Left: components right out of the printer (cell caps, cell body and detector holders). Right: ELG detail. 
The flow-through transmission cell was realized by SLA fabrication in tinted methacrylate resin (Formlabs Grey Standard). The monolithic body houses the $15 \mathrm{~mm}$ optical path, the fluidic ports, for a total volume inferior to $35 \mathrm{ul}$ (included connections). Path-lengths, detectors and sources are customizable and easily interchangeable, thanks to push-in optical connections (Figure 3). The optical windows feature an embedded light guide (ELG), a light-pipe integrated in the finished assembly, yielding a seamless joint at the fluid interface. The resulting finished components were pressurised and tested up to 3 bar and exhibited no sign of leakage. The ELG represents the core of cost savings and of increased performance, as it suppresses the need to purchase expensive off-the-shelf optical glass or fused quartz flow-cells or optical windows. Additionally, the ELG guarantees a precise and reproducible beam alignment, as the caps are registered directly into their respective recesses on the cell body. Any misalignment in the coupling of the sources and detector is cancelled, as light is homogenized and aligned right before and after passing through the optical path (Figure 4).

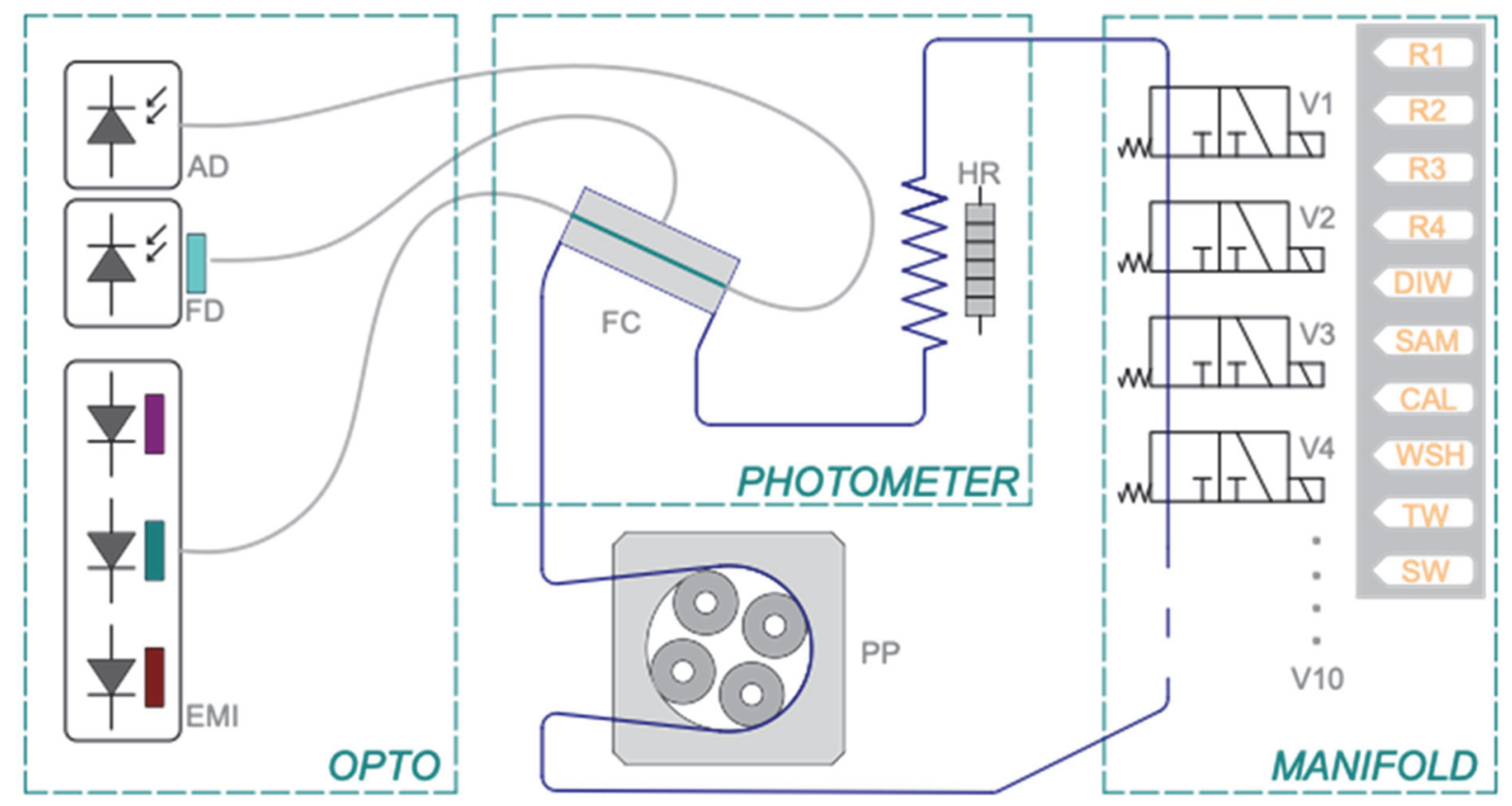

Figure 4. Opto-fluidic schematic. HR: heating resistor; FC: flow-cell; PP: peristaltic pump; V1-V10: solenoid valves 1 to 10; EMI: LED emitter section with BP filters; FD: fluorimetric mode detector; AD: absorbance mode detector; R1-R4: reagents 1 to 4; DIW: de-ionised water; SAM: sample port; CAL: calibration buffer; WSH: cleaning solution; TW-SW: toxic waste and safe waste discharge ports.

The emitter side features three LED sources with optional $10 \mathrm{~nm}$ FWHM bandpass filters (Knight Optical), which are multiplexed using a 3-to-1 custom multimode fibre optic combiner and then fibre-optic coupled to the cell. The detector is a light-to-frequency converter (TAOS-AMS) with spectral sensitivity ranging from 300 to $1100 \mathrm{~nm}$, peaking at around $690 \mathrm{~nm}$, which shows excellent linearity and great thermal stability.

Electronics and control

LEDs generally exhibit significant intensity drifts and minor wavelength shifts associated with environmental changes in temperature and/or self-heating, resulting in one the most common causes of instability in photometric detection. Each LED emitter is current regulated using the microprocessor on-board 12-bit DAC and a current pump, in steps of $0.1 \mathrm{~mA}$ from 0 to $25 \mathrm{~mA}$ (Figure 5). This allows the microprocessor to automatically adjust sources intensity during each analysis cycle, independently of sample turbidity and temperature, thus eliminating any non-linearity of the electronics and specifically of the detector, which is always kept in the same and reproducible operating point. Continuous dark correction is performed during each measurement many times per second (20 to 50 times per second), allowing the system to operate in noisy and changing environments, without the need for light shields. The measurements are then validated by detecting light instability between mixing steps, which is commonly associated with the presence of gas bubbles in the optical path. The development of the reaction is monitored continuously, and the measurement is performed once an established absorbance plateau is detected. This setup also allows for fixed time, kinetics, endpoint, and differential techniques to be performed. The resulting $\mathrm{CV}$ for 10 consecutive optical readings was generally found to be in the order of $0.1 \%$ for sample blank, and slightly higher after color development. 


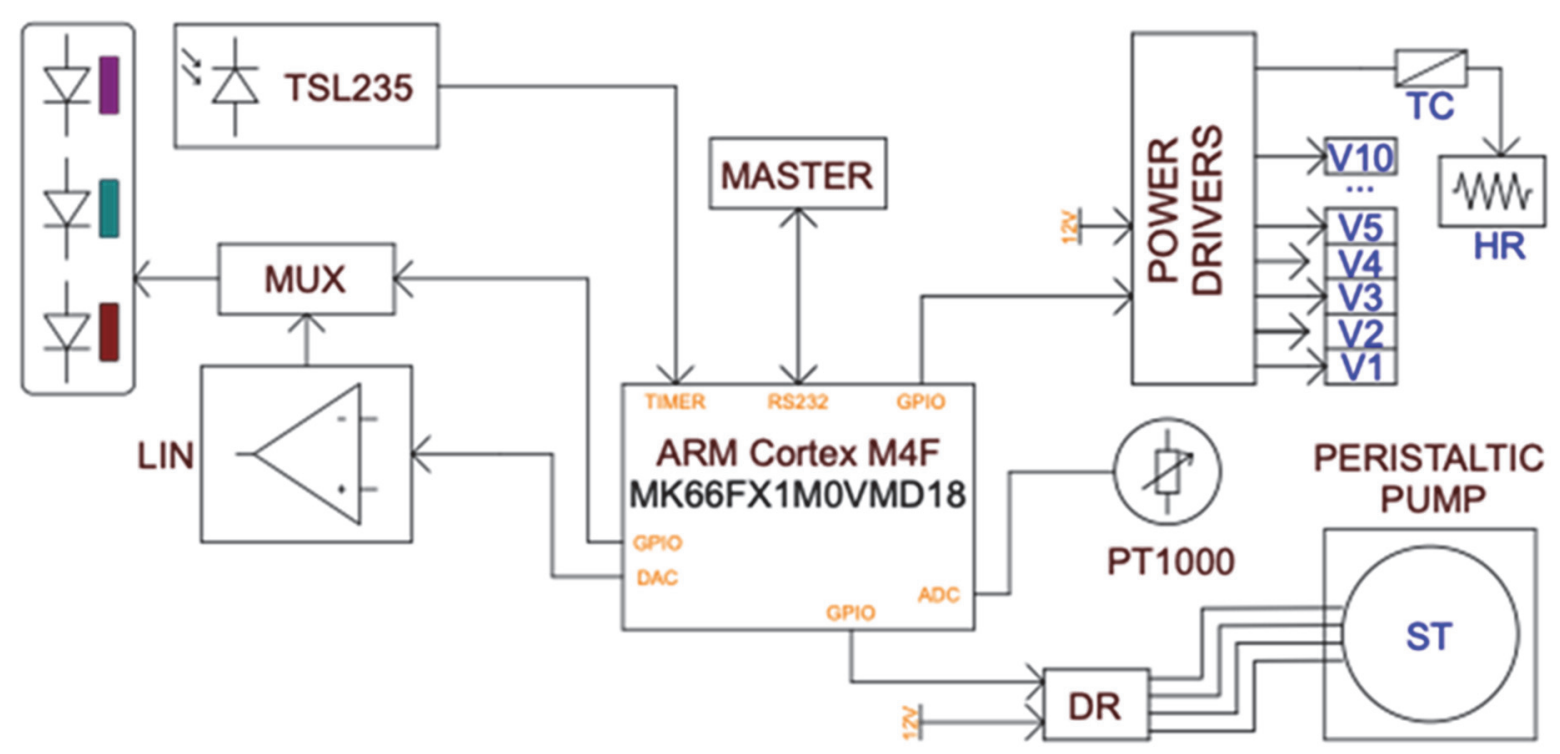

Figure 5. Electronics block diagram

\section{CALIBRATION}

The one or two-point auto-calibration is performed automatically by injecting into the loop one or more standard solutions. A sample calibration curve, instead, is shown in Figure 6, composed of 6 calibration points of 7 repetitions each, and generated autonomously by the analyser. It is relative to the vanadate/ascorbic acid method for phosphate measurement according to APHA Standard Method 4500-P-E: ammonium molybdate and potassium antimonyl tartrate (R1) will produce, in presence of orthophosphate and an acidic medium, phosphomolybdic acid, which, by means of ascorbic acid (R2), is in turn reduced to molybdenum blue, which is finally read at $880 \mathrm{~nm}$.

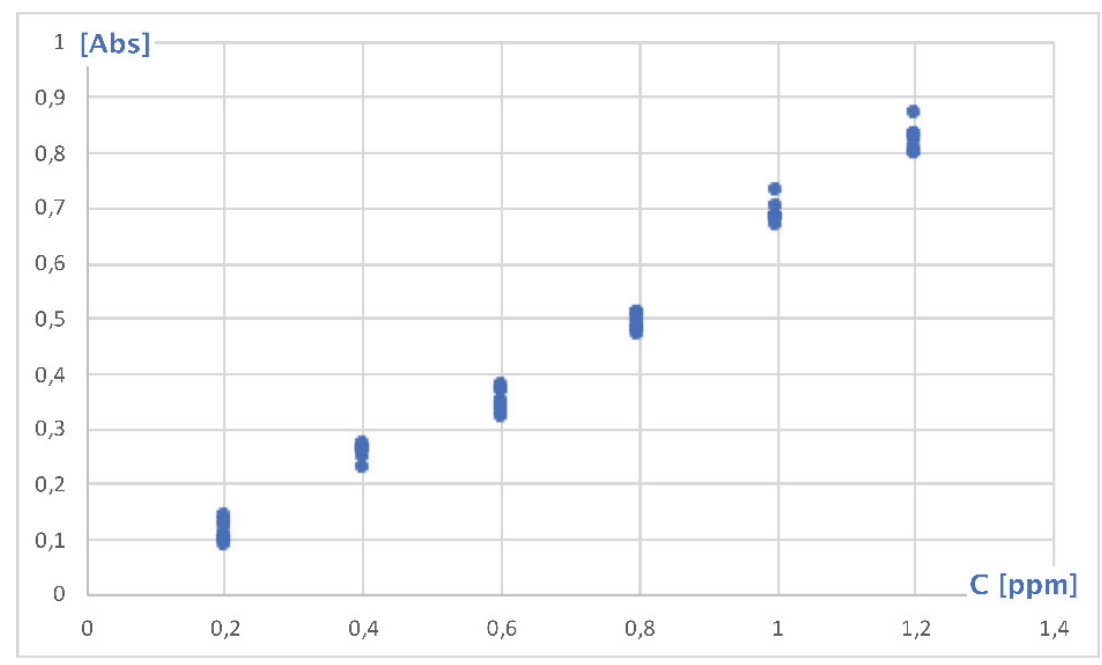

Figure 6. Phosphate APHA 4500-P E calibration at 6 different concentrations.

\section{CONCLUSION}

This short analysis of the uLIA analyser will hopefully serve as a proof of concept with regards to how additive manufacturing can ease and enhance the design of fluidic systems. By balancing the selection between commercial and custom-made components, the cost and performance of these systems can be tailored to a diverse range of environmental monitoring needs. Also, the progressive tendency towards the integration of diverse technologies and sub-systems into single monolithic and multi-functional components will further reduce both the cost and the physical footprint of these platforms, while increasing their reliability. Again, the use of additive manufacturing proves itself as a smart tool for easing and combining such a specialised integration level with a highly modular and scalable design. 


\section{ACKNOWLEDGEMENTS}

More information about the uLIA analyser is available at dptechnology.jimdo.com.

\section{REFERENCES}

Heinz E, Kraft P, Buchen C, Frede HG, Aquino E, Breuer L (2014) Set Up of an Automatic Water Quality Sampling System in Irrigation Agriculture. Sensors, 14, 212-228.

Howden NJK, Burt TP, Worrall F, Whelan MJ, Bieroza M (2010) Nitrate concentrations and fluxes in the River Thames over 140 years (1868-2008): are increases irreversible? Hydrol. Process., 24, $2657-2662$. doi:10.1002/hyp.7835

Mellander PE., Jordan P, Shore M, Melland AR, Shortle G (2015) Flow paths and phosphorus transfer pathways in two agricultural streams with contrasting flow controls, Hydrol. Process., 29, 3504-3518.

Mills G, Fones G (2012) "A review of in situ methods and sensors for monitoring the marine environment", Sensor Review, Vol. 32 No. 1, 17-28

Perks MT, Owen GJ, Benskin CMH, Jonczyk J, Deasy C, Burke S, Reaney SM, Haygarth PM (2015) Dominant mechanisms for the delivery of fine sediment and phosphorus to fluvial networks draining grassland dominated headwater catchments, Sci.Total Environ., 523, 178-190.

Trojanowicz M, Kołacińska K (2016) Recent advances in flow injection analysis. Analyst, 141(7), 2085-2139. https://doi.org/10.1039/C5AN02522B 